Research Article

\title{
Modelling the Dependency between Inflation and Exchange Rate Using Copula
}

\author{
Charles Kwofie (iD, Isaac Akoto, and Kwaku Opoku-Ameyaw \\ Department of Mathematics and Statistics, University of Energy and Natural Resources, Sunyani, Ghana \\ Correspondence should be addressed to Charles Kwofie; charles.kwofie@uenr.edu.gh
}

Received 26 March 2020; Revised 12 May 2020; Accepted 30 May 2020; Published 17 June 2020

Academic Editor: Aera Thavaneswaran

Copyright ( $\odot 2020$ Charles Kwofie et al. This is an open access article distributed under the Creative Commons Attribution License, which permits unrestricted use, distribution, and reproduction in any medium, provided the original work is properly cited.

In this paper, we propose a copula approach in measuring the dependency between inflation and exchange rate. In unveiling this dependency, we first estimated the best GARCH model for the two variables. Then, we derived the marginal distributions of the standardised residuals from the GARCH. The Laplace and generalised $t$ distributions best modelled the residuals of the GARCH $(1,1)$ models, respectively, for inflation and exchange rate. These marginals were then used to transform the standardised residuals into uniform random variables on a unit interval $[0,1]$ for estimating the copulas. Our results show that the dependency between inflation and exchange rate in Ghana is approximately $7 \%$.

\section{Introduction}

Macroeconomic variables such as inflation and exchange rate are very fundamental in any country's economy. They are the key indicators of performance of an economy as a whole. Several studies have focused on showing the dynamics and dependencies between these macroeconomic variables. According to the literature, inflation is of high priority to central banks because it gives an indication of price stability in an economy. Many argue that having high inflation leads to lower savings of individuals and also downplays an economy's international competitiveness. On the other hand, it is believed that a low inflation rate promotes economic growth. Some ways in which governments control inflation is by targeting exchange rates, interest rate dynamics, and others.

The literature somehow provides different results when it comes to the kind relationship between inflation and other macroeconomic variables such as the exchange rate. These conflicting results in the relationship between inflation and exchange rate differ with countries and data periods. Hence, the exchange rate can be said to be linked to inflation rate volatility through importation of goods and materials needed for production. The dependency of macroeconomic indicators has been shown in several papers to have some existing relationship [1-3]. However, this relationship has been unveiled using different approaches and methods. For instance, Kwofie and Ansah [4] used the autoregressive distributed lag (ARDL) model, while Arslaner et al. [1] used Markov switching regression and vector autoregression (VAR) methods in establishing some dynamics between inflation and exchange rate.

We are motivated by the work of Barro and Gordon [5] who pioneered and proposed an inflation and exchange rate nexus in relation to credibility of the monetary policy. Their argument was that any economy with a stable or fixed exchange rate regime has the tendency of lowering the inflation by authorities' increasing credibility. This assertion was echoed in $[6,7]$. They both stated that having a stable currency is not just a good step for maintaining inflation but also enhances monetary policy efficiency.

Anthony and Nkegbe [8] studied the relationships between several macroeconomic variables in Ghana using cointegration techniques. Their results showed a significant relationship between inflation and exchange rate in Ghana. Similarly, Gyebi and Boafo [9] stated in their conclusion that the exchange rate and money supply are the main macroeconomic variables responsible for inflation changes in the Ghanaian economy. Nortey et al. [10] stated that one of the main aims of Ghana's central bank is to maintain stability in 
the general level of prices in Ghana, and one way of achieving this is through exchange rate stability.

There have been numerous works in the literature that try to establish the relationship between inflation and exchange rate. Some of these works are $[11,12]$. In $[11,12]$, the authors analysed the relationship between inflation and exchange rate for some emerging markets, whereas Arslaner et al. [1] analysed a similar relationship using data from Turkey. Choudhri and Hakura [2] also analysed the relationship between inflation and exchange rate using data from several industrialised and developing countries. It is, however, important to note that all these authors used some form of multivariate time series analysis in unveiling the relationships. We, however, employ the copula-based modelling approach to analyse the relationship. In this approach, we combined both time series and probabilistic approach. The dependency between macroeconomic variables is a very important piece of information for policy making and enhancement. In this paper, we demonstrate how the copula-based approach can be used in modelling the relationship between inflation and exchange rate. The copula links two marginal distributions into a multivariate distribution, thereby providing some dependency parameters between them. We heavily rely on the famous theorem of Sklar [13]. This theorem basically states that any continuous multivariate distribution can be factored uniquely into its margins and a copula.

Our work is similar but different from [3] which also used copula to model the relationship between inflation and exchange rate using data from the European Central Bank between the period 2000 and 2016. The authors did this by using SARIMA to obtain the margins for the copula. However, our data were best fitted by $\operatorname{GARCH}(1,1)$ after which we fitted marginal distributions from the residuals and then obtained the uniform margins from these univariate distributions for copula estimation. Hence, it is a known fact that inflation and exchange rate are related. Several works are still being performed to understand the extent of the relationship that exists between these two variables. A work by Nkoro and Uko [14] in Nigeria was done to examine the relationship between inflation, exchange rate, and stock returns and was concluded that $\operatorname{GARCH}(1,1)$ correctly models these variables.

Ait Hassou et al. [3] used copula to model the dependency between inflation and exchange rate using data from European banks. Our study differs from this study in three ways:

(1) They used SARIMA, whilst we used GARCH for estimating the margins. First of all, our data exhibited no seasonal variation. Secondly, GARCH is known to capture volatility better than ARIMA, and from the plot of our data, one can easily see that our data exhibit high volatility.

(2) Our methodology was applied to data from a different time period and from a developing country, making it worthwhile to analyse.
(3) The methodologies are also different because they used a nonparametric approach, whilst we used a semiparametric approach.

The remaining part of the paper is organised under sections 2-4. Materials and methods for this work is presented in Section 2. This section consists of sections 2.1 (data), 2.2 (GARCH model), 2.3 (theory behind copula), 2.4 (distribution of marginals), 2.5 (copula models used), and 2.6 (estimation of parameters). We then present the results and discussion in Section 3. This section also consists of sections 3.1 (preliminary analysis), 3.2 (margins), and 3.3 (estimated copula). Lastly, the conclusion of the work is presented in Section 4.

\section{Materials and Methods}

Here, we describe and explain the data and the statistical methodology that were employed in unveiling the dependency structure that is expected between our variables of interest. In particular, we describe the GARCH model and the copula models that were used in evaluating the dependencies.

2.1. Data. We used monthly inflation and exchange rate data (Ghana cedi against the US dollar) from the Central Bank of Ghana. It comprised data points from the year 2000 to 2018.

2.2. GARCH Model. In identifying the structure of dependency, we first modelled each of our time series data with the generalised GARCH model developed in [15]. The bestselected model was selected by using the R software based on the AIC and BIC criteria. The residual of each model then enters into the next phase of deriving the marginals. Given any time series $\left(X_{i}\right)_{i \in Z}, \operatorname{GARCH}(p, q)$ can be expressed by

$$
\begin{aligned}
X_{i} & =\sigma_{i} \epsilon_{i}, \\
\sigma_{i}^{2} & =\alpha_{0}+\sum_{j=1}^{p} \alpha_{j} \epsilon_{i-j}^{2}+\sum_{k=1}^{q} \beta_{k} \sigma_{i-k}^{2},
\end{aligned}
$$

where $\left\{\epsilon_{i}\right\}_{i \in \mathbb{N}}$ are iid with $\mathbb{E}\left(\epsilon_{i}\right)=0$ and $\operatorname{Var}\left(\epsilon_{i}\right)=1$ and the model parameters $\alpha_{j}, j=0, \ldots, p$ and $\beta_{k}, k=0, \ldots, q$ are such that $\alpha_{j}, \beta_{k} \geq 0$ with $\sum_{j=1}^{p} \alpha_{j}+\sum_{k=1}^{q} \beta_{k} \leq 1$.

2.3. Theory behind Copulas. The use of copulas got momentum in economics and finance only in the last few years, and the example works are [16-18]. In our study, we make use of two variables, and hence a brief discussion of bivariate copula theory is given in the following. The discussion is largely taken from Goorbergh [19]. We first provide a statistical definition of a copula and then state Sklar's theorem with its probabilistic interpretation, and then we discuss the relationship between copulas and their dependence measures.

Definition 1. A copula function in two dimensions is $C:[0,1]^{2} \longrightarrow[0,1]$ which satisfies:

(1) $C(u, v)=C(0, v)=0$ for every $u, v \in[0,1]$ 
(2) $C(u, 1)=u$ and $C(1, v)=v$ for every $u, v \in[0,1]$

(3) $C\left(u_{2}, v_{2}\right)-C\left(u_{2}, v_{1}\right)-C\left(u_{1}, v_{2}\right)+C\left(u_{1}, v_{1}\right) \geq 0$ for every $u, v \in[0,1]$

Theorem 1 (Sklar's theorem, see [13]). Suppose F and G are marginals with a joint distribution $H$. Then, a copula $C$ exists with

$$
H(x, y)=C(G(x), F(y)),
$$

$\forall(x, y) \in \mathbb{R} \times \mathbb{R} . C$ is unique given that $F$ and $G$ are continuous; otherwise, the copula $C$ is determined uniquely using Rank F $\times$ Rank G. The converse of 2.2 also holds true.

The proof of the above theorem can be found in [20]. The theorem shows that any joint distribution can be decomposed into its marginal distributions and a copula, which describes the dependence between the marginals. Conversely, it states that given any marginals and a copula, we can combine them to obtain a joint distribution.

A widely used dependence measure in copulas in measuring dependence is Kendall's tau. For any two random variables $X$ and $Y$ with Copula $C$, their Kendall's tau can be expressed as

$$
\tau_{C}=4 \iint_{[0,1]^{2}} C(u, v) d C(u, v)-1,
$$

for $u, v \in[0,1]$.

2.4. Distribution of Marginals. We assume that the realisation of the standardised residuals from our $\operatorname{GARCH}(\mathrm{p}, \mathrm{q})$ model for both variables follows any one of the following marginal distributions, where $f_{Y}(y)$ is the probability density function (pdf) of the marginals.

\subsubsection{Generalized t Distribution}

$$
f_{Y}(y)=\psi\left\{2 \sigma \omega^{1 / \psi} \mathbf{B}\left(\frac{1}{\psi}, \omega\right)\left[1+\frac{|z|^{\psi}}{\omega}\right]^{\omega+(1 / \psi)}\right\}^{-1},
$$

for $\mu, y \in \mathbb{R}, \sigma, \omega, \psi \in \mathbb{R}^{+}, z=(y-\mu) / \sigma$, and $\mathbf{B}(\cdot, \cdot)$ is the beta function given by

$$
\mathbf{B}(\alpha, \beta)=\int_{0}^{1} t^{\alpha-1}(1-t)^{\beta-1} \mathrm{~d} t, \quad \text { for } \alpha, \beta>0 .
$$

\subsubsection{Skew Normal Distribution}

$$
f_{Y}(y)=2 \phi(z) \Phi(\beta z) ; y, \beta, \mu \in \mathbb{R}, \sigma \in \mathbb{R}^{+},
$$

where $z=y-\mu / \sigma, \phi(\cdot)$ is the probability density function (pdf) of the standard normal distribution given by $f_{X}(x)=(1 / \sqrt{2 \pi}) \exp \left\{-x^{2} / 2\right\}, x \in(\infty, \infty)$, and $\Phi(\cdot)$ is its cumulative distribution function (cdf) given by $F_{X}(x)=1 / 2[\operatorname{erf}(x / \sqrt{2})+1], x \in(-\infty, \infty)$.

\subsubsection{Laplace Distribution}

$$
f_{Y}(y)=\frac{1}{2 \tau} \exp \left\{-\frac{|y-\mu|}{\tau}\right\},
$$

for $y, \mu \in \mathbb{R}$ and $\tau \in \mathbb{R}^{+}$.

\subsubsection{Logistic Distribution}

$$
f_{Y}(y)=\frac{1}{t} \exp \left\{\frac{y-n}{t}\right\}\left(1+\exp \left\{\frac{y-n}{t}\right\}\right)^{-2}
$$

for $t \in \mathbb{R}^{+}$and $y, n \in \mathbb{R}$.

These four distributions were used due to their easy adaptability to copula modelling. They also have widespread use in modelling distributions of macroeconomic data such as inflation and exchange rate.

2.5. Copula Models Used. Here, we describe the copulas that were used in this work. In this paper, the relationship between inflation and exchange rate in Ghana was modelled by two bivariate copulas. We used the elliptical and the Archimedean family of copulas for modelling. The description together with their Kendall's tau can be found in Table 1. The last two which is the Gaussian and Student $t$ copulas make up the elliptical family, while the other eight make up the Archimedean family.

2.6. Estimation of Parameters. Given that our marginal densities $g($.$) and f($.$) have parameters \theta_{1}$ and $\theta_{2}$, respectively, whilst that of the copula depends on $\theta_{3}$. Now, suppose that we have a given data sample pair $\left(x_{i}, y_{i}\right), i=$ $1, \ldots, n$ of size $n$, then the log-likelihood of the joint distribution can be written as

$$
\begin{aligned}
L\left(\theta_{1}, \theta_{2}, \theta_{3}\right):= & \sum_{i=1}^{n} \log g\left(x_{i} ; \theta_{1}\right)+\sum_{i=1}^{n} \log f\left(y_{i} ; \theta_{2}\right) \\
& +\sum_{i=1}^{n} \log C\left(G\left(x_{i} ; \theta_{1}\right), F\left(y_{i} ; \theta_{2}\right) ; \theta_{3}\right),
\end{aligned}
$$

where $C($.$) is the copula to be estimated. From this log-$ likelihood function, the parameters of our copula can be estimated.

\section{Results and Discussion}

This section deals with the results obtained by applying the methodology to our inflation and exchange rate data. We first obtain some descriptives and visualisation of our data which we call preliminary analysis. Then, we estimate the univariate margins using GARCH. After that we derive the marginal probability distribution of the standardised residuals, and then transform them to get our uniform marginals which we then use to estimate the copulas.

3.1. Preliminary Analysis. We present some descriptive statistics and a plot of our variables. As already mentioned, 


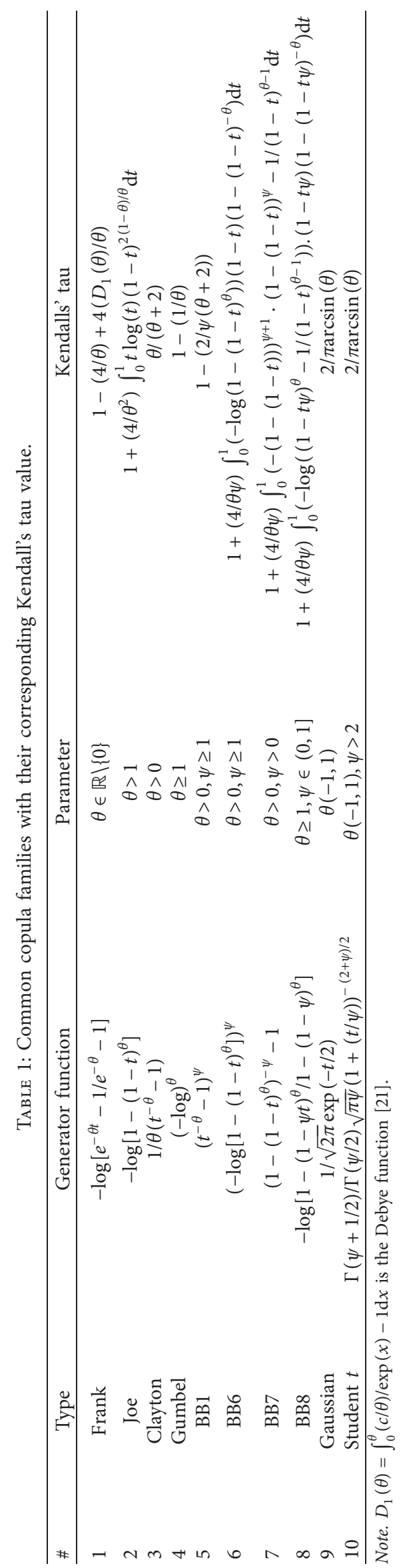




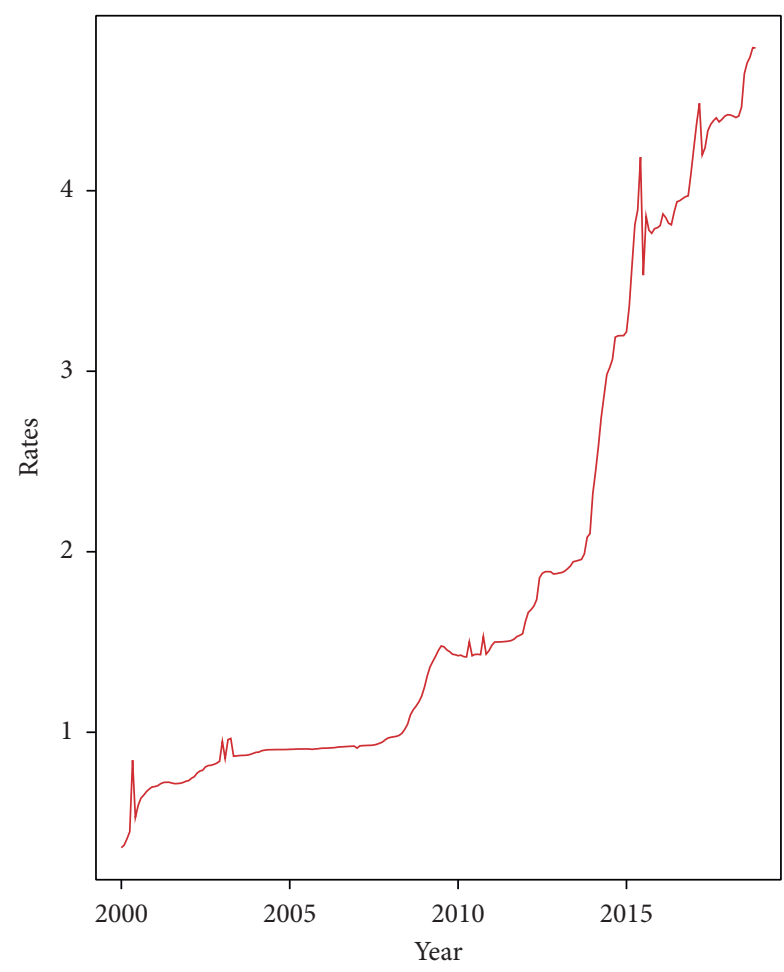

(a)

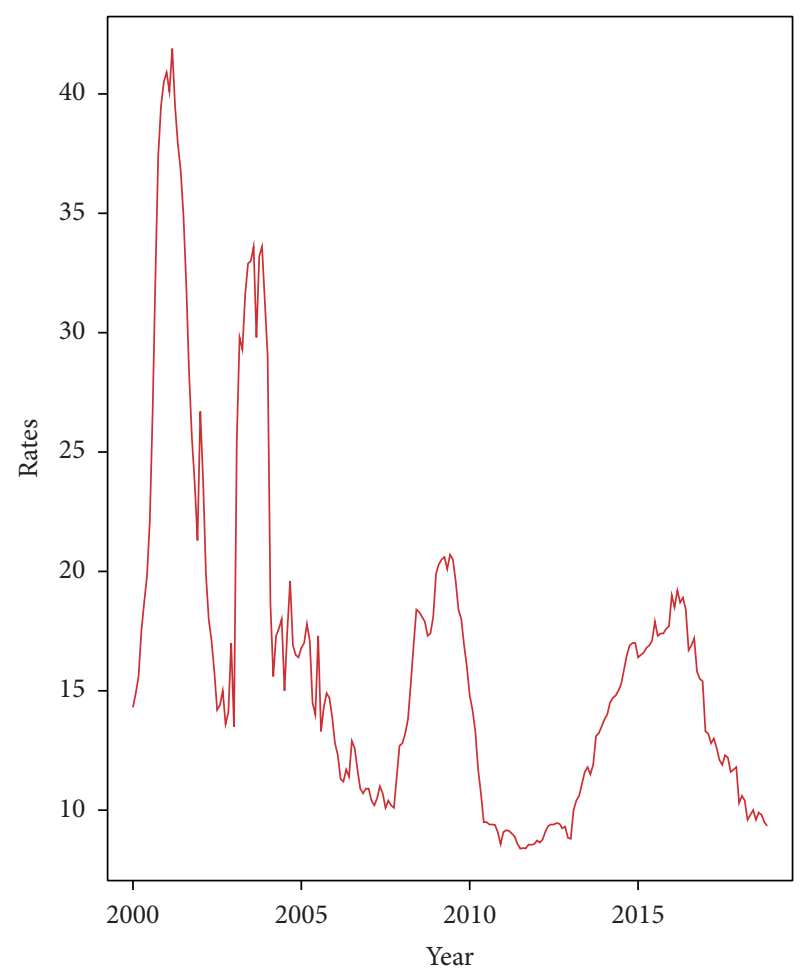

(b)

FIgUre 1: Plot of (a) monthly exchange rates (USD) of Ghana and (b) monthly inflation rates of Ghana.

we use monthly data of inflation and exchange rate from the Bank of Ghana, spanning from 2000 to 2018. The plot clearly depicts that the variables were not stationary. Hence, the subsequent analysis of data was carried out using the first difference of both variables.

Figure 1 shows the time series plot of both monthly inflation and exchange rate data. Clearly, the plot shows a highly recognizable volatility which supports our choice for using GARCH for modelling the univariate margins. The standard deviation of inflation rates of 7.89 in Table 2 confirms the high spikes in the plot seen in Figure 1.

3.2. Margins. We present the best-suited GARCH model for our dataset in Tables 3 and 4 . The best-fitted GARCH model for both inflation and exchange rate was $\operatorname{GARCH}(1,1)$. The results for both variables show that all the parameters of both models were significant at 5\% significance level with the exception of the constant in the exchange rate model. From the GARCH models, we retrieve the standardised residuals $\left(x_{e}\right)$ and fit marginal distributions for each dataset before we transform the fitted marginals into uniform marginal distributions for building our copula. Table 5 shows that the standardised residuals are not normally distributed, and this result is consistent with what the financial time literature says.

The uniform marginals are obtained by transforming the standardised residuals from our GARCH model using the best-fitted marginal distributions. So, now, we fit probability distributions to the standardised residuals of each of our
TABle 2: Descriptives.

\begin{tabular}{lcc}
\hline Statistic & Inflation rate & Exchange rate (USD) \\
\hline Mean & 16.35 & 1.84 \\
Standard deviation & 7.48 & 1.30 \\
Minimum & 8.39 & 0.36 \\
Maximum & 41.90 & 4.79 \\
\hline
\end{tabular}

TABLE 3: Estimated coefficients of the GARCH $(1,1)$ model for the exchange rate.

\begin{tabular}{lcccc}
\hline & Estimate & Std. error & $t$ value & $P$ value \\
\hline$\alpha_{0}$ & $1.679 e-06$ & $3.792 e-04$ & 0.004 & 0.996 \\
$\alpha_{1}$ & $8.619 e-02$ & $1.638 e-02$ & 5.262 & $1.42 e-07$ \\
$\beta_{1}$ & $1.744 e-01$ & $8.404 e-02$ & 2.075 & 0.038 \\
LLF & 1.702745 & & & \\
BIC & 0.11059599 & & & \\
\hline
\end{tabular}

TABle 4: Estimated coefficients of the GARCH $(1,1)$ model for inflation.

\begin{tabular}{lcccc}
\hline & Estimate & Std. error & $t$ value & $P$ value \\
\hline$\alpha_{0}$ & $1.5270 e-02$ & $5.4850 e-03$ & 2.784 & $0.00537^{* *}$ \\
$\alpha_{1}$ & $9.4359 e-02$ & $1.5047 e-02$ & 6.271 & $3.58 e-10^{* * *}$ \\
$\beta_{1}$ & $1.4623 e-02$ & $8.8895 e-03$ & 1.644 & 0.10018 \\
LLF & -631.7143 & & & \\
BIC & 5.661358 & & & \\
\hline
\end{tabular}


TABLE 5: Testing for normality of our $\operatorname{GARCH}(1,1)$ standardised residuals.

\begin{tabular}{lcc}
\hline \multirow{2}{*}{ Data } & \multicolumn{2}{c}{ Normality } \\
& Jarque-Bera & Shapiro-Wilk \\
\hline Inflation & 0.008 & 0.000 \\
Exchange rate & 0.0071 & 0.00127 \\
\hline
\end{tabular}

TABLE 6: Marginal distribution of our inflation rate residuals.

\begin{tabular}{lcc}
\hline Distribution & AIC & BIC \\
\hline Logistic & 501 & 512 \\
Skew normal & 377 & 362 \\
Generalised $t$ & 257 & 312 \\
Laplace & 112 & 141 \\
\hline
\end{tabular}

TABLe 7: Marginal distribution of our exchange rate residuals.

\begin{tabular}{lcc}
\hline Distribution & AIC & BIC \\
\hline Logistic & 230 & 251 \\
Skew normal & 201 & 215 \\
Generalised $t$ & 119 & 128 \\
Laplace & 189 & 191 \\
\hline
\end{tabular}

fitted GARCH model. Using AIC and BIC values, it can be seen in Tables 6 and 7 that the best-fitted marginals for the residuals of the GARCH models of inflation and exchange rate were, respectively, the Laplace and generalised $t$ distributions. We now transform these probability distributions into uniform random variables on the unit interval $[0,1]$. This was done using the well-known idea that a random variable composed with itself gives a uniform random variable. This uniform variable derivation and the transformation on the unit interval $[0,1]$ were simply done with the $\mathrm{R}$ software. To confirm that our transformations are uniformly distributed on the unit interval $[0,1]$, we conduct a formal statistical test. The result of this test is presented in Table 8 . The result shows that they are uniformly distributed on the unit interval $[0,1]$. For the estimation of our copula, it is essential that we check the dependency between our new variables. The result is presented in Table 9. It shows that the two variables are dependent.

3.3. Estimated Copulas. We now present the result of the estimation of the parameters of our selected copulas. The parameters were estimated in $\mathrm{R}$ software using maximum likelihood estimation. The Gaussian, Student $t$, Clayton, Gumbel, Joe, BB1, Frank, BB6, BB7, and BB8 copulas were estimated, and the results are presented in Table 10. Each of these copula parameters was statistically significant at $5 \%$ significance level, indicating some significant statistical dependency between inflation and exchange rate.

The Kendall's $\tau$ for each copula was used to measure the dependence between inflation and exchange rate. The table shows that the dependency between inflation and exchange rate is approximately $7 \%$.
TABLE 8: Uniformity test on the interval $[0,1]$.

\begin{tabular}{lcc}
\hline Margins & Statistic & $P$ value \\
\hline$u_{1}$ & 0.0571 & 0.2120 \\
$u_{2}$ & 0.0812 & 0.2751 \\
\hline
\end{tabular}

TABLe 9: Test of independence between marginals using Kendall's rank correlation.

\begin{tabular}{lcc}
\hline Pair & $\tau$ & $P$ value \\
\hline$u_{1}, u_{2}$ & 0.20612 & 0.214 \\
\hline
\end{tabular}

TABLE 10: Estimated copulas' parameter estimates and goodnessof-fit criteria values.

\begin{tabular}{lccccc}
\hline \multirow{2}{*}{ Copula } & \multicolumn{2}{c}{ Parameter } & \multicolumn{2}{c}{ Goodness of fit } & \multirow{2}{*}{ Kendall's $\tau$} \\
& $\theta$ & $\psi$ & BIC & LLF & \\
\hline Gaussian & 0.06 & & 4.66 & 0.38 & 0.073 \\
Student $t$ & 0.07 & 4.5 & -8.28 & 9.57 & 0.072 \\
Clayton & 0.1 & & 5.43 & 0 & 0.071 \\
Gumbel & 1 & & 5.43 & 0 & 0.071 \\
Frank & 0.27 & & 0.36 & 4.71 & 0.070 \\
Joe & 1.2 & & 5.43 & 0 & 0.070 \\
BB1 & 0.12 & 2.1 & 10.89 & -0.02 & 0.077 \\
BB6 & 1 & 1 & 10.90 & -0.02 & 0.079 \\
BB7 & 0.2 & 0.5 & 10.87 & -0.01 & 0.079 \\
BB8 & 1 & 0 & 10.79 & 0 & 0.072 \\
\hline
\end{tabular}

\section{Conclusion}

In conclusion, we showed that copulas can be used in measuring the dependency between inflation and exchange rate data. We used monthly inflation and exchange rate data for this study. We found out that the Laplace distribution and the generalised $t$ distribution best modelled inflation and exchange rate, respectively, in Ghana. According to the goodness-of-fit test for the various copulas, it was discovered that Student $t$ best captured the dependency between our variables. The result from our best-fitted copula (Student $t$ ) shows that the dependency between inflation and exchange rate is approximately $7 \%$. This implies that to target inflation, one of the key indicators to tackle is the appreciation or the depreciation of the Ghana cedi against the US dollar. The result of this study is consistent with a lot of studies on the two variables in Ghana using different approaches. Kwofie and Ansah [4], for example, stated that there is some relationship between them in their paper. More generally, our result shows that exchange rate stability can be used to achieve a more stable inflation to some extent. However, there could as well be many other factors that could drive inflation.

\section{Data Availability}

The data for this work are readily available on the website of the Bank of Ghana. 


\section{Conflicts of Interest}

The authors declare that there are no conflicts of interest.

\section{References}

[1] F. Arslaner, D. Karaman, N. Arslaner, and S. Hilmi Kal, "The relationship between inflation targeting and exchange rate pass-through in Turkey with a model averaging approach," Central Bank of the Republic of Turkey Working Paper, vol. 1, no. 14/16, 2014.

[2] E. U. Choudhri and D. S. Hakura, "Exchange rate passthrough to domestic prices: does the inflationary environment matter?" Journal of International Money and Finance, vol. 25, no. 4, pp. 614-639, 2006.

[3] L. Ait Hassou, F. Badaoui, O. G. Cyrille, A. Amine, A. Zoglat, and E. Ezzahid, "Copulas for modeling the relationship between inflation and exchange rate," in Proceedings of the International Work-Conference on Time Series Analysis, Springer, Granada, Spain, pp. 217-228, September 2017.

[4] C. Kwofie and R. K. Ansah, "A study of the effect of inflation and exchange rate on stock market returns in Ghana," International Journal of Mathematics and Mathematical Sciences, vol. 2018, Article ID 7016792, 8 pages, 2018.

[5] R. J. Barro and D. B. Gordon, "A positive theory of monetary policy in a natural rate model," Journal of Political Economy, vol. 91, no. 4, pp. 589-610, 1983.

[6] R. Dornbusch, "Fewer monies, better monies," American Economic Review, vol. 91, no. 2, pp. 238-242, 2001.

[7] A. Velasco, "Fixed exchange rates: credibility, flexibility and multiplicity," European Economic Review, vol. 40, no. 3-5, pp. 1023-1035, 1996.

[8] C. Anthony and P. K. Nkegbe, "GDP growth, money growth, exchange rate and inflation in Ghana," Journal of Contemporary Issues in Business Research, vol. 3, no. 2, pp. 75-87, 2014.

[9] F. Gyebi and G. K. Boafo, "Macroeconomic determinants of inflation in Ghana from 1990-2009," International Journal of Business and Social Research, vol. 3, no. 6, pp. 81-93, 2013.

[10] E. N. N. Nortey, D. D. Ngoh, K. Doku-Amponsah, and $\mathrm{K}$. Ofori-Boateng, "Modeling inflation rates and exchange rates in Ghana: application of multivariate garch models," SpringerPlus, vol. 4, no. 1, p. 86, 2015.

[11] F. G. Caselli and A. Roitman, "Nonlinear exchange-rate passthrough in emerging markets," International Finance, vol. 22, no. 3, pp. 279-306, 2019.

[12] M. Ca'Zorzi, E. Hahn, and M. Sánchez, Exchange Rate Passthrough in Emerging Markets, 2007.

[13] S. Sklar, Fonctions de Reprtition an Dimensions et Leursmarges, 1959.

[14] E. Nkoro and A. K. Uko, "Exchange rate and inflation volatility and stock prices volatility: evidence from Nigeria, 1986-2012," Journal of Applied Finance and Banking, vol. 6, no. 6, p. 57, 2016.

[15] T. Bollerslev, "A conditionally heteroskedastic time series model for speculative prices and rates of return," The Review of Economics and Statistics, vol. 69, no. 3, pp. 542-547, 1987.

[16] U. Cherubini, E. Luciano, and V. Walter, Copula Methods in Finance, John Wiley \& Sons, Hoboken, NJ, USA, 2004.

[17] A. J. Patton, "Modelling asymmetric exchange rate dependence," International Economic Review, vol. 47, no. 2, pp. 527-556, 2006.

[18] A. Charpentier, J.-D. Fermanian, and O. Scaillet, "The estimation of copulas: theory and practice," in Copulas: From
Theory to Application in Finance, J. Rank, Ed., pp. 35-64, Risk Books, London, UK, 2007.

[19] R. Van den Goorbergh, "A copula-based autoregressive conditional dependence model of international stock markets," Technical Report, Netherlands Central Bank, Research Department, Amsterdam, Netherlands, 2004.

[20] R. B. Nelsen, An Introduction to Copulas, Springer Science \& Business Media, Berlin, Germany, 2007.

[21] E. Brechmann and U. Schepsmeier, "Modeling dependence with c-and d-vine copulas: the R package CDVine," Journal of Statistical Software, vol. 52, no. 3, pp. 1-27, 2013. 